\title{
Inheritance of reduction, loss, and asymmetry of the pelvis in Pungitius pungitius (ninespine stickleback)
}

\author{
D. M. BLOUW \& G. J. BOYD* \\ Department of Biology, St Francis Xavier University, Antigonish, Nova Scotia, Canada B2G 1 CO and *Faculty of \\ Medicine, Dalhousie University, Halifax, Nova Scotia, Canada B3H $4 H 7$
}

\begin{abstract}
The pelvis of Pungitius pungitius (ninespine stickleback) is normally a robust, bilaterally symmetrical structure with stout spines. In some populations it is reduced in size, sometimes to complete absence. The first sign of reduction is loss of the spines, which is accompanied by a reduction in the size of the pelvic bones (vestiges) and an increase in bilateral asymmetry. Further reduction in the size of the vestiges leads to their eventual loss. In a pelvis-reduced population the propensity to spine asymmetry is highly heritable $\left(h^{2}=0.85 \pm 0.14\right)$ but asymmetry of the bony vestiges in fish without spines is not heritable. The absence of spines is heritable, as is size of the bony vestiges $\left(h^{2}=0.49 \pm 0.12\right)$. Crosses of spineless fish from a reduced population with spined fish from either of two normal populations produce only spined progeny. The inter-population heritability of pelvis size is $h^{2}=0.26 \pm 0.10$, but that of bilateral asymmetry is zero. The expression of spines and vestiges is influenced relatively little by variation in $\mathrm{pH}$, calcium, and salinity. We propose a polygenic model of inheritance with two phenotypic thresholds, the upper for the presence of spines and the lower for complete absence of the pelvis. Canalization breaks down between the thresholds. This genetic system probably predates divergence of the stickleback genera. We discuss its relevance to understanding the dynamics of pelvis reduction in extant and fossil stickleback populations.
\end{abstract}

Keywords: bilateral asymmetry, heritability, pelvis reduction, Pungitius, stickleback, threshold trait.

\section{Introduction}

Reduction and loss of the pelvis occurs in three genera of stickleback fishes (Gasterosteidae: Culaea, Gasterosteus and Pungitius) and it occurs throughout the boreal region (Nelson \& Atton, 1971; Stephanidis, 1971; Coad, 1973; Moodie \& Reimchen, 1973; Campbell, 1979; Giles, 1983; Edge \& Coad, 1983; Takata \& Goto, 1985; Bell et al., 1985a). However, in spite of its widespread occurrence the phenomenon is rare; most populations of all species show the normal condition of a well-developed pelvis with stout spines (Nelson, 1971a). Reduction is considered to be a derived condition (Bell, 1974, 1987) and it is hypothesized to have arisen independently in the three genera and among widely separated populations within species (Nelson, 1977; Bell, 1984, 1987).

Reduction is nearly always associated with loss of the pelvic spines. When present the spines are thought to function as a mechanical defense against predators, in agonistic display to territorial rivals and intruders, and in courtship display (Hoogland et al. 1957; Wootton, 1976, 1984). Hence spine loss is expected to reduce both the survival and reproductive components of fitness. Nevertheless the frequency of reduced phenotypes is high and apparently stable over time in some extant populations (Nelson, 1977; Reimchen, 1980; Reist, 1981; McPhail, in press), and it persisted for over 90,000 years in a Miocene stickleback, Gasterosteus doryssus (Bell et al., 1985b).

The factors that enable maintenance of reduced phenotypes in extant populations are unknown, although several hypotheses have been advanced Nelson, 1969; Reimchen, 1980; Reist 1980a,b; Giles, 1983). Tests of these hypotheses are hampered, however, because although there is evidence that the reduction is heritable (Nelson, 1977; Campbell, 1979; Giles, 1983), its genetic basis remains unknown.

Our purpose is to investigate the inheritance of pelvic reduction and loss in Pungitius pungitius using 
intra- and inter-population crosses reared under controlled laboratory conditions. Fish from our pelvisreduced populations show high levels of bilateral asymmetry in the pelvis and we also estimate its heritability.

\section{Materials and methods}

\section{Pelvis phenotypes}

In normal, unreduced, populations of Pungitius the pelvis is bilaterally symmetrical and consists of two pelvic spines supported on a ventral shield (Fig. 1, complete). The shield has anterior, ascending, and posterior processes, and is well ossified. Reduction involves loss of the pelvic spines and diminished size of the bones of the ventral shield (Fig. 1, incomplete). Individuals with total loss have neither spines nor vestigial elements of the shield (Fig. 1, absent). The variation is not a smooth series but has discontinuities, first

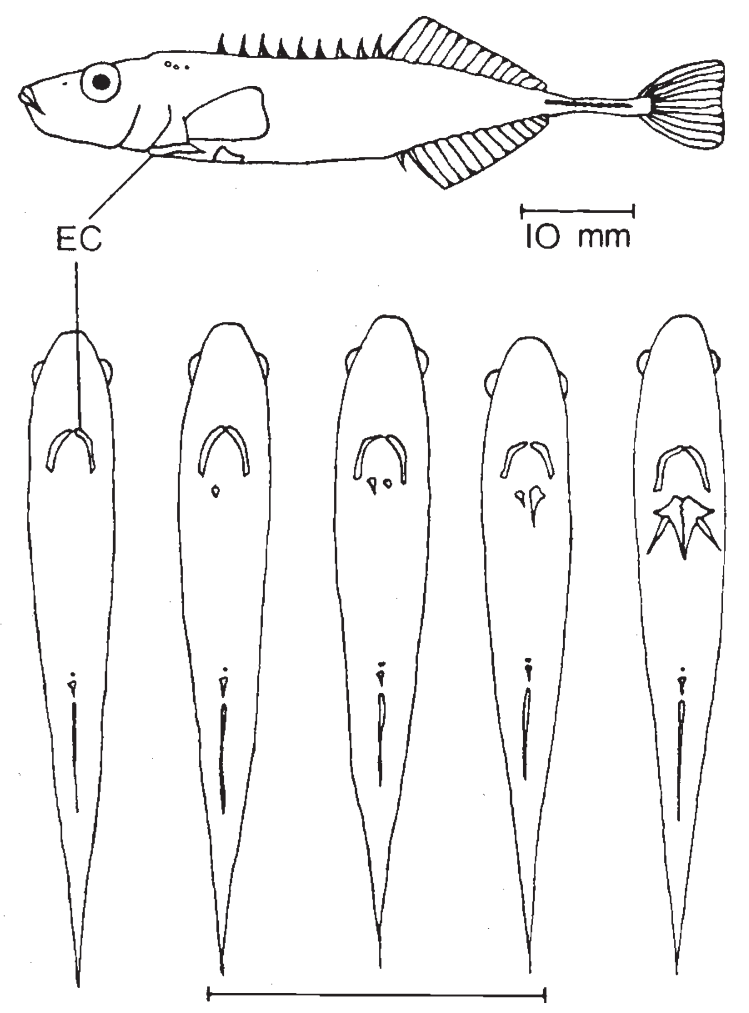

Absent Incomplete Complete

Fig. 1 Semi-diagrammatic representation of the range of pelvis variability in Pungitius of O'Keefe's Lake, Prince Edward Island, Canada. The ectocoracoid bone (EC) is shown as a landmark. Complete fish have pelvic spines and good bilateral symmetry. Incomplete fish have no spines, the size of the pelvic bones is variable and reduced, and bilateral asymmetry is much greater than in complete fish. There is no trace of the pelvis in specimens scored as absent. with loss of spines and second with loss of all elements of the pelvis. There is an abrupt reduction in the size of the pelvic bones when spines are lost and the bilateral halves exhibit pronounced asymmetry.

Because the pelvis is a complex of components we scored the parts separately. Spines were scored as present or absent on each side. Development of the pelvis was scored by measuring the sizes of constituent bones. They are irregular in fish that show pelvic reduction so the size was recorded as the greatest antero-posterior dimension of the bilateral halves. The difference between right and left was used to estimate bilateral asymmetry, and the sum was used to estimate total size. The sum is independent of asymmetry considerations while still reflecting the overall pelvis development. Pelvis size was standardized to a common body length of $42.5 \mathrm{~mm}$, the grand mean size of all fish used in the study; intra-population regression coefficients from the parental population samples were used to standardize parental phenotypes and the overall regression coefficients of offspring (across all families, or subsets of families as appropriate) were used to standardize offspring phenotypes. The effects of differences in body size were also controlled statistically by analysis of covariance (ANCOVA), with body length as the covariate.

All specimens were stained with Alizarin Red S to ensure that even the smallest calcified vestiges were identified and scored. Measurements were made with a dissecting microscope and ocular micrometer. Fish length was measured from the tip of the snout to the end of the hypural plate. We have evidence from sequential sampling of progeny in some of our crosses and from field samples of young fish from normal and reduced populations that the pelvis is fully formed by the time the fish are $25 \mathrm{~mm}$ long. In any case we scored no fish smaller than $30 \mathrm{~mm}$.

\section{Source populations}

As with other sticklebacks, pelvis reduction and loss occurs throughout the distribution of Pungitius (Nelson, 1971b; Stephanidis, 1971; Coad, 1973; Takata and Goto, 1985). The population we used occurs in O'Keefe's Lake in central Prince Edward Island, Canada. Fewer than 2 per cent of the O'Keefe's Lake fish have a fully formed pelvis, about 70 per cent have a reduced pelvis and it is absent in the remainder. O'Keefe's Lake is isolated, with neither inlet nor outlet streams, it is acidic $(\mathrm{pH}<6)$ and there is little calcium present $\left(<1.5 \mathrm{mg}^{-1}\right)$.

Inter-population crosses of O'Keefe's Lake fish were made with Pungitius from Glenfinnan River, a nearby $(10 \mathrm{~km})$ population. Because the results from O'Keefe's 
Lake $\times$ Glenfinnan River crosses were unexpected we made four additional inter-population crosses using parents from a distant $(200 \mathrm{~km})$ population, Black River, New Brunswick. In large samples $(n>250)$ we observed no pelvis reduction at either Glenfinnan River or Black River.

\section{Husbandry and rearing conditions}

Gravid females and mature males were collected from source populations during the breeding season (May-July) and maintained in 400-litre aquaria at $15-17^{\circ} \mathrm{C}$ under an extended photoperiod (8D:16L) in the laboratory. Mortalities during transport and holding were less than 3 per cent and both sexes remained in active reproductive condition throughout the holding period. Offspring were reared uniformly at $15-17^{\circ} \mathrm{C}$ at a photoperiod of $10 \mathrm{D}: 14 \mathrm{~L}$.

Field observations on euryhaline sticklebacks suggest that the ionic environment might influence pelvic expression, with greater skeletal development at higher salinity, $\mathrm{pH}$, and calcium, concentrations (McPhail, 1963; Wootton, 1976, 1984; Giles, 1983; Bell, 1984). To evaluate the importance of these environmental variables we reared progeny in brackish water of two salinities (4 ppt and $14 \mathrm{ppt}$ ), with high calcium concentrations $\left(>14 \mathrm{mg} 1^{-1}\right)$, and neutral $\mathrm{pH}$ $(7.4 \pm 0.5)$. If there is environmental modification of the phenotype this rearing regime should produce progeny with strongly developed skeletal structures. With the exception of an ionic environment, we followed standard stickleback crossing and husbandry practices (Hagen, 1973; Bell, 1984).

\section{Results}

\section{Comparison of the parental populations}

The contrast between the O'Keefe's Lake and Glenfinnan River populations is illustrated in the two upper panels of Fig. 2. In O'Keefe's lake fewer than 2 per cent of the fish have pelvic spines (complete) and about 30 per cent have no vestige of the pelvis (absent), whereas all fish from Glenfinnan River have a fully formed pelvis. The size of the supporting bones is much less in O'Keefe's Lake than in Glenfinnan River (Fig.2), even in the few fish with one or more spines.

Asymmetry in the parental populations was examined by testing whether the (right-left) differences in antero-posterior length of the pelvic elements had a mean of zero $(t$-test) and a normal distribution (Lilliefors test and normal probability plots; for rationale see Palmer \& Strobeck, 1986). We excluded fish without a pelvis as they are perfectly symmetrical by default.

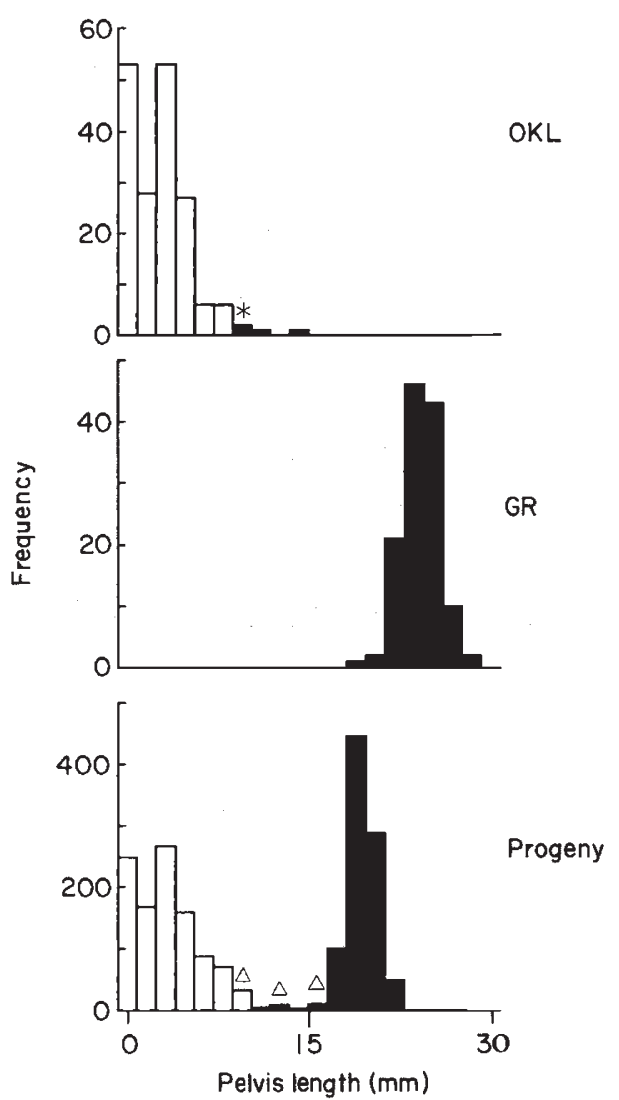

Fig. 2 Distribution of pelvis lengths among spined (- $)$ and spineless ( $(\square)$ fish from the two major source populations (OKL= O'Keefe's Lake, GR = Glenfinnan River) and in the $F_{1}$ progeny. The distribution of pelvis lengths for the Black River population is similar to that of Glenfinnan River. In the panel for progeny those without spines are the product O'Keefe's Lake × O'Keefe's Lake intra-population crosses while those with spines are largely from inter-population crosses. Pelvis length is expressed as the sum of the bilateral halves. Fish with a pelvis length of zero are excluded. In the column marked * one fish has no spines, and in those marked with a triangle there is a mixture of spined and spineless fish. Data for the source populations were scored from fish that were sampled and preserved when parental stocks were collected in the field.

Neither the means of O'Keefe's Lake nor those of Glenfinnan River differ significantly from zero (Fig.3), which suggests that the asymmetry is not directional, however, neither distribution is normal, which suggests antisymmetry (disproportionate development occurring at roughly equal frequencies on either side, characterized by platykurtic or bimodal distributions of asymmetry values; Palmer \& Strobeck, 1986). The distribution of Glenfinnan River, however, is leptokurtic with a slight negative skew (Fig. 3). We conclude that the Glenfinnan River population shows fluctuating asymmetry which is tightly constrained about the mean of zero. 


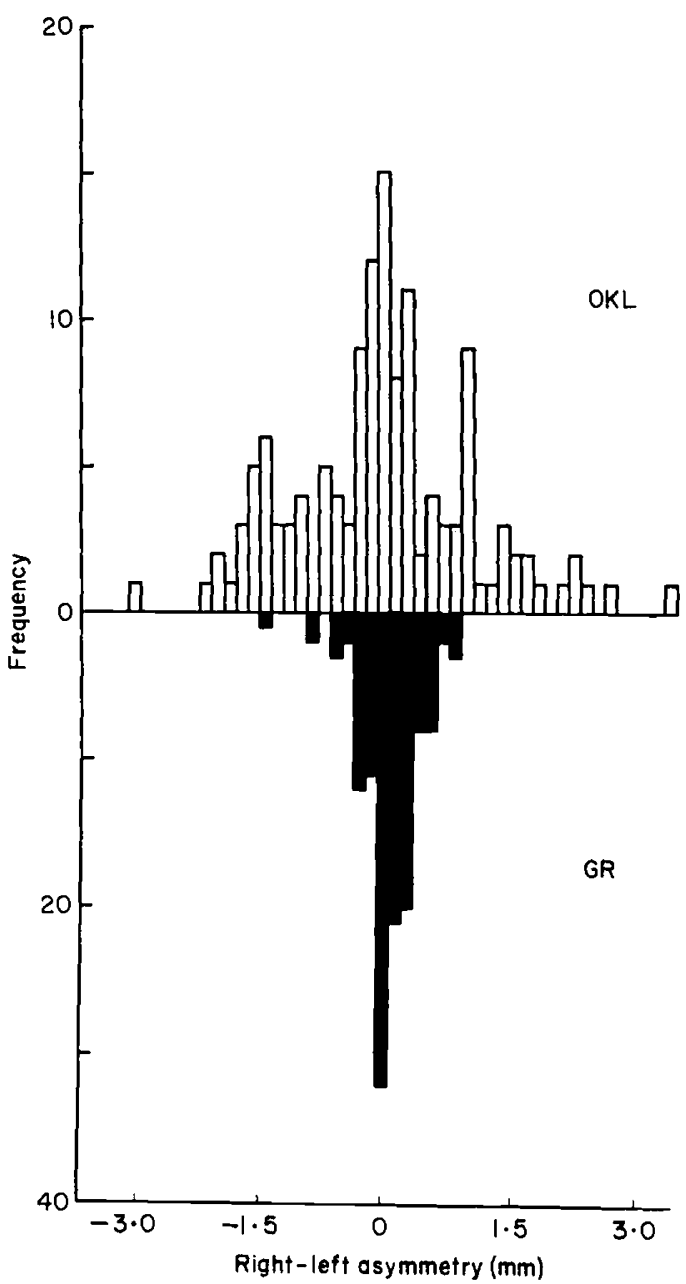

Fig. 3 Distribution of pelvis asymmetry values in the O'Keefe's Lake (OKL) and Glenfinnan River (GR) parental populations. All fish without a pelvis are excluded because they are symmetrical by default.

In contrast, the distribution of O'Keefe's Lake asymmetries is platykurtic and the asymmetry, estimated by variance in the (right-left) differences, is much greater than in Glenfinnan River (Fig. 3; ANCOVA, $P<0.001$ ). Therefore the O'Keefe's Lake population shows elevated bilateral asymmetry, which may reflect both fluctuating asymmetry and antisymmetry. Since fish from O'Keefe's Lake without a pelvis were excluded and very few have spines, then it is clear that the pronounced asymmetry in this population must be due to differential development of the vestiges of incomplete fish.

\section{O'Keefe's Lake intra-population crosses - asymmetry}

The high level of asymmetry in O'Keefe's lake fish is of concern because it suggests that there is an unstable relationship between genotype and phenotype. As a consequence we investigated whether the asymmetry is heritable, first in terms of spines, and secondly in terms of the size of vestiges.

We scored fish as symmetrical if they had either no spines or both spines, and asymmetrical if only one spine was present. Asymmetrical males were used as parents in seven O'Keefe's Lake intra-population crosses (three left, four right). No asymmetrical females were used. Progeny of asymmetrical males are more often asymmetrical than expected (Table 1a; contingency $\chi^{2}=52.3, P<0.001$ ). The narrow sense heritability and its standard error, estimated by assuming a polygenic threshold model (method 2 of Falconer, 1965 ), is $h^{2}=0.846 \pm 0.136$. Although we used only asymmetrical males, the asymmetrical progeny are two males and nine females, hence there is no evidence of sex linkage or sex-limited expression. Furthermore, as shown in Table $1 \mathrm{~b}$, there is no evidence that the direction of asymmetry is heritable in the 11 asymmetrical progeny from asymmetrical parents. We conclude that the propensity to asymmetry in spines is highly heritable and that spine asymmetry is fluctuating.

The inheritance of asymmetry in vestiges was evaluated by offspring-midparent regression. Because there is no single index of asymmetry that is clearly superior to others, we made estimates using: (i) simple (right-left) differences, (ii) the absolute value of (right-left) differences, (iii) the variance of (right-left)

Table 1 (a). Segregation of pelvic spine phenotypes among progeny of symmetrical and asymmetrical parents from O'Keefe's Lake. Symmetrical fish have either no spines or both spines. Only male parents were asymmetrical; all female parents were without spines. Expected values (contingency $\left.\chi^{2}\right)$ are shown in parentheses. (b). Segregation of pelvic spines among the 11 asymmetrical progeny of asymmetrical parents in (a).

\begin{tabular}{lll}
\hline & \multicolumn{2}{l}{ Progeny } \\
\cline { 2 - 3 } (a) Parents & Symmetrical & Asymmetrical \\
\hline Symmetrical & 938 & 8 \\
& $(928.8)$ & $(17.2)$ \\
Asymmetrical & 89 & 11 \\
& $(98.2)$ & $(1.8)$ \\
\hline & Progeny & \\
\cline { 2 - 3 } & Left spine & Right spine \\
\hline (b) Parents & 6 & 2 \\
\hline Left spine & 2 & 1 \\
Right spine & &
\end{tabular}


differences (Palmer \& Strobeck, 1986). Analyses were made using families in which neither parent had spines, and excluding all spined progeny.

There is no statistically significant relationship between asymmetry in parents and their progeny using any of the three indices of asymmetry. The heritability remains zero when estimates are made on male and female parents separately, and/or when progeny with no vestiges (symmetrical by default) are excluded. Therefore, in contrast to spine asymmetry, we conclude that vestige asymmetry is not heritable and that it represents developmental noise.

\section{O'Keefe's Lake intra-population crosses}

We first consider the presence/absence of spines, then the size of the pelvic vestiges. We scored fish as either having $(1$ or 2$)$ or not having spines $(0)$. Segregation of spined and spineless progeny from the two types of parents is shown in Table 2; there is a highly significant $\left(\chi^{2}=137.87 ; P<0.001\right)$ excess of spined progeny from spined parents. The narrow sense heritability, again estimated by method 2 of Falconer (1965), is $h^{2}=1.154 \pm 0.107$. This estimate, greater than the expected maximum of 1 , is clearly inflated. It may be that a major gene is involved and that the polygenic model is inappropriate, however no simple Mendelian model fits the data and presumptive homozygotes under Mendelian models segregate inappropriate progeny. The inflated estimate is more likely to be due to rearing offspring in an ionic environment (neutral $\mathrm{pH}$, elevated salinity and calcium) which is expected to accentuate skeletal development. The results are clearly consistent with the conclusion that spine loss is highly heritable.

Vestiges in incomplete fish show continuous size variation therefore we used offspring-midparent regression (Falconer, 1981) to estimate heritability. To avoid threshold effects we excluded all crosses with

Table 2 Segregation of spined and spineless progeny in crosses among both types of parents from O'Keefe's Lake. Spined fish have one or both spines. Expected values (contingency $\chi^{2}$ ) are shown in parentheses.

\begin{tabular}{lcc}
\hline & \multicolumn{2}{c}{ Progeny } \\
\cline { 2 - 3 } Parents & Spineless & Spined \\
\hline Spineless & 924 & 5 \\
& $(905.02)$ & $(23.98)$ \\
Spined & 95 & 11 \\
& $(113.98)$ & $(3.02)$ \\
\hline
\end{tabular}

spined parents, all crosses with both parents having no pelvis, all spined progeny, and all progeny with no pelvis. The regression slope is significant $(P<0.01)$ and the narrow sense heritability and standard error are $h^{2}=0.49 \pm 0.12$. Thus vestige size is heritable, while vestige asymmetry (above) is not.

\section{Inter-population crosses}

The progeny of O'Keefe's Lake and Glenfinnan River crosses all have a complete pelvis except for three (of 870 ) that have one spine instead of two. All progeny from O'Keefe's Lake $\times$ Black River crosses have a complete pelvis. Hence when the pelvic-reduced fish of O'Keefe's Lake are crossed with normal fish from either adjacent (Glenfinnan River) or distant (Black River) populations the progeny are normal. The pelvis size of inter-population progeny (O'Keefe's Lake $\times$ Glenfinnan River or Black River) is significantly greater than that of the O'Keefe's Lake $X$ O'Keefe's Lake progeny (Fig. 3; ANCOVA, $P<0.001$ ). However the hybrid progeny have a smaller pelvis than the Glenfinnan River parental population (Fig. 3; $P<0.001$ ), which indicates that although the pelvis is complete, the influence of O'Keefe's Lake parentage is nevertheless detectable. The inter-population heritability of pelvis size, estimated by offspring-midparent regression, is $h^{2}=0.26 \pm 0.10$.

There is no significant difference in bilateral asymmetry between the Glenfinnan River parental population and O'Keefe's Lake $\times$ Glenfinnan River inter-population progeny $(P>0.05$; ANCOVA $)$, but the inter-population progeny are far more symmetrical than the O'Keefe's Lake $\times$ O'Keefe's Lake intrapopulation progeny $(P<0.001)$. Finally, there is no relationship between the asymmetry of parents and progeny using any of the three indices of asymmetry (above), so we conclude that the propensity to asymmetry is not heritable in inter-population crosses.

\section{Influence of rearing environment}

The pelvis size of offspring reared at high salinity is not significantly greater $(P>0.05)$ than that of fish reared at lower. However pelvis size is slightly but significantly $(P<0.05$, ANCOVA $)$ larger in laboratory-reared O'Keefe's Lake $\times$ O'Keefe's Lake progeny than in the O'Keefe's Lake field sample and, as shown above, more spined progeny than expected under a threshold model segregated from crosses with one spined parent (Table 2) in the laboratory. Both observations might be attributable to the ionic composition of the rearing environment. Bilateral asymmetry does not differ significantly between the O'Keefe's Lake population and 
the O'Keefe's Lake $\times$ O'Keefe's Lake progeny, nor between the Glenfinnan River population and the O'Keefe's Lake $\times$ Glenfinnan River progeny. Thus environmental modification of the phenotype does occur but its influence seems minor.

Although the mean asymmetry of all progeny pooled does not differ significantly from zero, nevertheless within a number of individual families there is a significant directional bias. Among O'Keefe's Lake $\times$ O'Keefe's Lake crosses, in which asymmetry is high, only two of 49 families show bias, which is expected by chance alone (Type 1 error). In contrast, although the level of asymmetry is much less, six of 18 O'Keefe's Lake × Glenfinnan River families show signi-

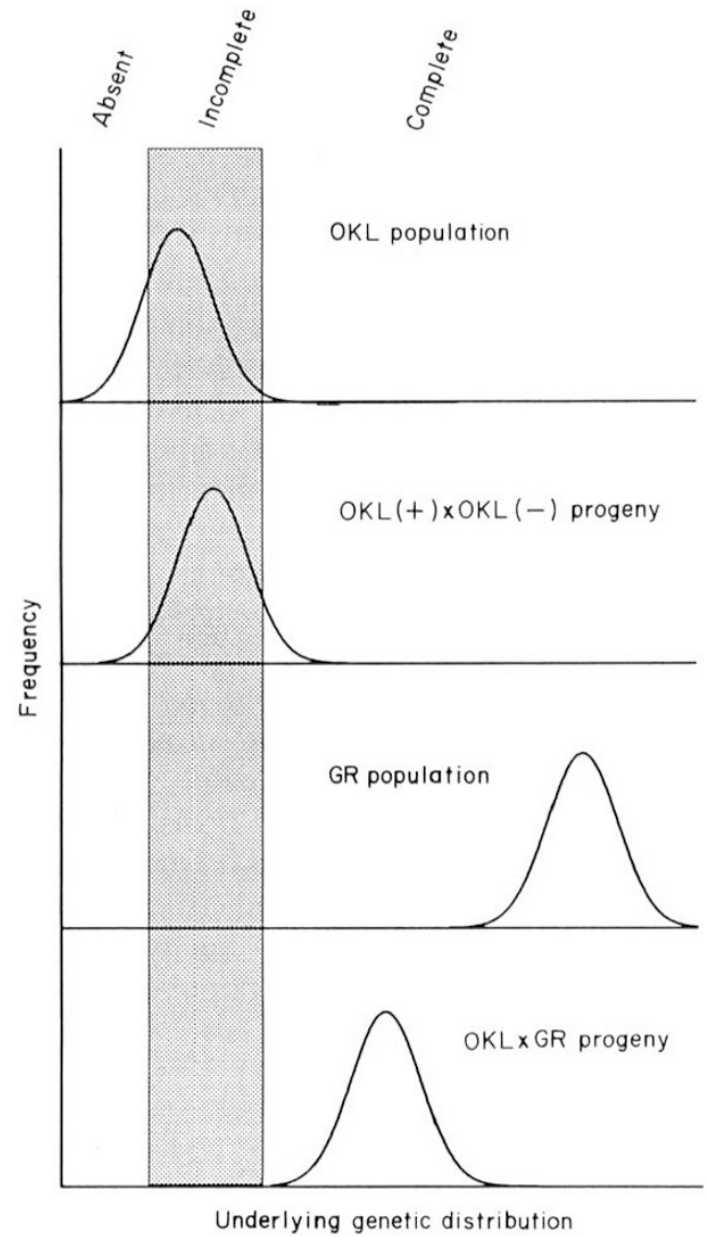

Fig. 4 Threshold model of pelvis inheritance. Abbreviations as in previous figures; OKL $(+)$ indicates spined fish and $\mathrm{OKL}(-)$ spineless fish. Genotypes in the shaded area give rise to an incomplete pelvis and high bilateral asymmetry. Fish above the shaded area have a complete pelvis with spines, and those below have no trace of the pelvis. Therefore the margins of the shaded area represent phenotypic thresholds, the upper for presence/absence of spines, and the lower for presence/absence of any trace of the pelvis. See text (Discussion) for further details. ficant bias, three to the left and three to the right. We do not observe directional asymmetry in O'Keefe's Lake $\times$ Black River progeny but family sizes are too small to enable confident conclusions. The reason for increased directional bias in inter-population crosses, where the overall level of asymmetry is lower, is unclear.

\section{Discussion}

Our results show that pelvis reduction is heritable in Pungitius, and they are consistent with a model of polygenic inheritance with two thresholds. The upper threshold is for loss of spines (incomplete), and the lower for absence of the pelvis (absent). Above the upper threshold and between thresholds the size of the pelvis behaves as a simple quantitative trait. However, whereas canalization is well established above the upper threshold, it is disrupted between thresholds as shown by increased bilateral asymmetry of vestiges. Hence the presence of spines and vestiges demarcate phenotypic thresholds while the size of the pelvis is more reflective of the continuous underlying genetic distribution.

We hypothesize with this model that the O'Keefe's Lake population has a genetic distribution relative to thresholds as shown in the upper panel of Fig. 4. The O'Keefe's Lake intra-population crosses, in which we used spined males as parents, are then equivalent to directional selection and the expected response of an increased number of progeny with spines is observed as shown in the second panel (Fig. 4). The Glenfinnan River and Black River populations are hypothesized to have genetic distributions far above the upper threshold (third panel, Fig. 4) as phenotypically manifested in the large size of the pelvis (Fig. 3). Their interpopulation progeny with O'Keefe's Lake parents therefore lie closer to the threshold (lower panel, Fig. 4) and, consistent with a polygenic model, they have smaller pelvis bones (Fig. 2).

If this model is correct then predictable relationships should persist between parents and progeny with genotypes between the first and second thresholds - pelvis size should behave as a simple polygenic trait without the complication of discontinuous phenotypes. As shown above (Results), this is what we find ( $h^{2}$ of vestiges is $0.49 \pm 0.12$ ). We further expect in O'Keefe's Lake intra-population crosses that: (i) spineless progeny of spined parents should have a significantly larger pelvis than spineless progeny of spineless parents, (ii) spineless parents that produced spined progeny, should have a larger pelvis than spineless parents that do not produce spined progeny, (iii) there should be a deficiency of progeny with no trace 
of the pelvis in those crosses where one parent is spined relative to those where neither parent was spined, and (iv) there should be an excess of progeny with no trace of the pelvis from crosses where at least one parent has no trace of the pelvis. All these expectations are upheld in our dataset (analyses not shown), but of course they and other predictions should be tested against new data from independent crosses. The only evidence inconsistent with a threshold model is the heritability estimate over 1.0 for spine presence but this might be attributable to the altered rearing environment (Results).

Pelvis reduction has evolved independently in widely separated populations within each of the three extant genera of sticklebacks that occur in fresh water and it occurred in the Miocene Gasterosteus doryssus (Bell, 1988). It seems probable, therefore, that the genetic system underlying pelvis reduction also predates generic divergence and that it is common among the species and populations which currently exhibit the phenomenon. Campbell (1979) and Giles (1983) each made crosses with pelvis reduced Gasterosteus aculeatus from the Outer Hebrides, Scotland, and concluded that reduction has some heritable basis. In a more comprehensive study, Nelson (1977) made 16 laboratory crosses and some controlled field introductions with brook sticklebacks (Culaea inconstans). He scored pelvic phenotypes in three classes (absent - A, incomplete - I, complete - C). Although relatively few of the laboratory-reared progeny survived, over 75 per cent of the progeny of $C \times C$ crosses segregated as complete, and over 80 per cent of the progeny of $\mathrm{A} \times \mathrm{A}$ crosses segregated as absent. Approximately half the progeny of $\mathrm{A} \times \mathrm{I}$ crosses and $\mathrm{C} \times \mathrm{A}$ crosses segregated as absent. These results are consistent with a polygenic threshold model. Results from the field introductions are similar to those in the laboratory, however there may have been a mixing of populations due to flooding, and replication of $\mathrm{C} \times \mathrm{C}$ introductions in different years has given heterogeneous results. Given high (and potentially selective) mortality in the laboratory and variable results from the field, Nelson (1977) concluded that pelvis loss and reduction have some heritable basis.

Lande (1978) reviewed the genetic basis of limb and digit loss in tetrapods and concluded that reduction is either under polygenic control with thresholds or subject to polygenic modification. With thresholds, canalization between thresholds, and assumed constant heritability and selection coefficients, Lande (1978) showed that the rate of evolution is about six times faster when populations are centred on a threshold than when they are in a region of canalization between thresholds. This is because selection is most efficient when phenotypes are at intermediate frequencies and relatively inefficient when the phenotypic variance is low. Lande (1978) also showed that limb and digit reduction can evolve very rapidly, even with small selection coefficients.

That pelvis reduction in sticklebacks is in response to selection is well established (Reimchen, 1980; Reist, 1980a,b; Giles, 1983; reviewed in Bell, 1987, 1988). However the mechanism and even the target of selection remain unclear. The results of Reimchen (1980) and Reist $(1980 \mathrm{a}, \mathrm{b})$ implicate predation by fishes as selective for spines in mechanical defence, and predation by invertebrates as selective against spines, because the spines are hypothesized to provide holdfasts for grappling predators. These suspected agents may interact because fish prey on invertebrates as well as sticklebacks (Reimchen, 1980) so that the balance between fish and invertebrate predation may be a complex function of their relative abundances, the availabilities of alternative prey types, and the availability of refuge. Giles (1983) noted that $G$. aculeatus populations which exhibit pelvis reduction in Scotland coexist with a known predator (brown trout, Salmo trutta), but the reduction occurs in lakes with low calcium concentrations. Adjacent lakes with generally higher calcium levels support populations with a normal pelvis. Giles (1983) suggested that pelvis reduction in these populations is in response to selection for phenotypes with reduced calcium demands. Bell (1987) maintains that while there is evidence of directional selection for pelvis reduction where it occurs, nevertheless the morphology of vestiges is not subject to selection. Thus Bell (1987) implicitly argues that vestiges are non-functional and that the target of selection is something other than their form, perhaps skeletal reduction generally or absence of spines specifically.

With our genetic model we predict that directional selection for pelvis reduction by invertebrate predators should have different consequences from that of selection to reduce calcium demand. The target of selection by grasping invertebrate predators is any aspect of morphology which provides a more secure holdfast, whereas the target with low calcium concentrations is any structure which requires calcium uptake against steep concentration gradients from the environment (another target, presumably, would be any physiological attribute which enhances ability to sequester calcium). Grappling invertebrate predators therefore are hypothesized to be selective against projections from the body surface, and particularly those in the body plane exposed to attack. Bottom dwelling ambush predators, such as odonate larvae, will most frequently attack from below, where the pelvic spines are located. Such predators are then expected to exert directional 
selection for phenotypes below the upper threshold (Fig.4) but not beyond (unless, of course, the vestiges themselves provide a holdfast). This is in contrast to selection for reduced calcium demand which should remain effective throughout all phenotypic stages to complete absence, although the intensity of selection should diminish in proportion to size of the pelvis.

Furthermore, with selection for reduced calcium demand we expect a response in skeletal structures generally, with the greatest response in those structures that are least essential to fitness and that have the highest levels of phenotypic and genetic variance. In contrast, if the target of selection is spines only, then we do not expect a reduction in other skeletal elements (skull, vertebral column, fin rays, lateral plates), except through pleiotropy or genetic correlation. Only if genetic correlation with other skeletal elements is a pervasive component of the genetic system will spine reduction persistently be accompanied by skeletal reduction. If this is the case it will be difficult to distinguish the effects of selection by invertebrate predators from those due to reduced calcium. Clearly we need estimates of phenotypic and genetic correlations among skeletal traits and detailed analysis of covariation between phenotypes and environment.

Given polygenic inheritance with thresholds, the appropriate selective environments must be coupled with restricted gene flow for pelvis reduction to evolve in natural populations. This may explain why reduction tends to occur in isolated populations and in headwater lakes, where the opportunity for gene flow with individuals from normal populations is limited (Stephanidis, 1971; Coad, 1973; Moodie \& Reimchen, 1973; Campbell, 1979; Reimchen, 1980; Edge \& Coad, 1983; Giles, 1983; Takata \& Goto, 1985; McPhail, in press). Even in Alberta, Canada, in a large and ecologically diverse area where two genera (Pungitius and Culaea) show convergence in pelvis reduction (Nelson \& Atton, 1971; Nelson, 1971b; Reist, 1981), the reduced populations of both species tend to be genetically isolated.

Our genetic results allow insight into a sequence of Miocene fossil $G$. doryssus which provides a continuous 110,000 -year history of pelvis reduction in what was most likely a single population in a saline lake (Bell et al., 1985b; Bell, 1988). The pelvis was reduced at the beginning of the sequence but further gradual reduction took place over 93,000 years. Then a population with a fully formed pelvis appears in the deposit, it lived sympatrically with $G$. doryssus for $60-120$ years (however this time interval is at the limit of temporal resolution of the fossil sequence), and then replaced the reduced form (Bell, 1988). Over the following 3000 years pelvis reduction was re-established in the population. Bell et al. (1985b) note that there is a smooth phenotypic transition during this rapid reversion in which all intermediate forms are present. This rapid evolution is consistent with directional selection on a polygenic threshold trait with even modest selection coefficients (Lande, 1978). The same rapid evolutionary rate would be possible with even lower selection coefficients if interbreeding had occurred between the reduced $G$. doryssus and the invaders, rather than extinction of the pre-existing lineage and its replacement by the genetically independent invaders as suggested by Bell (1988). As heritability estimates become available for Gasterosteus (or assuming they are similar to those reported here), and with more precise estimates of the time course, it should be possible to estimate the intensity of selection that occured in this fossil lineage

Our results emphasize a need for caution in interpreting the biological meaning of bilateral asymmetry. Fluctuating asymmetry is often interpreted to reflect development instability (Allendorf \& Leary, 1986; Palmer \& Strobeck, 1986) yet, for this to be true, it is necessary that the asymmetry be environmental in origin, not genetic. In the case of pelvic spines the propensity to asymmetry is highly heritable, and asymmetrical fish (those with one spine) presumably have genotypes positioned near the upper threshold (Fig.4). Spine asymmetry therefore reflects genetic proximity to a threshold. Immediately below the threshold vestige asymmetry is not heritable, so the great asymmetry observed in bony vestiges of O'Keefe's Lake fish is probably a reliable indicator of the inability of the pelvis developmental system to buffer perturbation. Thus variation in the pelvis provides an example where asymmetry in one part of a complex structure (spines) poorly reflects developmental stability (rather reflecting proximity to a phenotypic threshold on the underlying genetic distribution), while that in another (vestiges) is probably true fluctuating asymmetry with no heritable basis.

Increased development instability, as reflected by fluctuating asymmetry, has been correlated with increased homozygosity, with the breakdown of coadapted development genetic systems in hybridization, and with stressful environmental extremes or dramatic alterations of the environment (Allendorf \& Leary, 1986; Palmer \& Strobeck, 1986). Pelvis asymmetry in sticklebacks is clearly associated with directional selection (increased homozygosity) and, because it happens rarely, we infer that the selection is engendered by unusual and possibly extreme environments. It is not a consequence of hybridization as shown by the similarity levels in parental and hybrid progeny in our inter-population crosses. 


\section{Acknowledgements}

We thank Betty (Miles) Gloutney for her talented dedication to the successful husbandry of thousands of sticklebacks, and for cheerfully scoring much of the material. The animated discussion and exuberant field assistance of Terese Hansen, Trish MacEachern, Larry MacPhee, and Steve Saxon is gratefully acknowledged. M. Bell, L. Blouw, and G. Haas made very useful comments on the manuscript. Financial support was through NSERC grant A2324 to DMB, an NSERC Undergraduate Scholarship to GJB, and by the St Francis Xavier University Council for Research.

\section{References}

ALLENDORF, F. W. AND LEARY, R. F. 1986. Heterozygosity and fitness in natural populations of animals. In: Soulé, M. E. (ed.) Conservation Biology. The Science of Scarcity and Diversity, Sinauer Assoc. Inc., Sunderland, MA.

BELL, M. A. 1974. Reduction and loss of the pelvic girdle in Gasterosteus (Pisces): A case of parallel evolution. Natural History Museum, Los Angeles Country, Contributions to Science, 257, 1-36.

BELL, M. A. 1984. Evolutionary phenetics and genetics: the threespine stickleback, Gasterosteus aculeatus, and related species. In: Turner, B. J. (ed.) Evolutionary Genetics of Fishes, Plenum Press, New York.

BELL, M. A. 1987. Interacting evolutionary constraints in pelvic reduction of threespine sticklebacks, Gasterosteus aculeatus (Pisces, Gasterosteidae). Biological Journal of the Linnean Society, 31, 347-382.

BELL, M. A. 1988. Stickleback fishes: Bridging the gap between population biology and paleobiology. Trends in Ecology and Evolution, 3, 320-325.

BELL, M. A., FRANCIS, R. C. AND havens, A. C. 1985a. Pelvic reduction and its directional asymmetry in threespine sticklebacks from the Cook Inlet Region, Alaska. Copeia, 1985, 437-444.

Bell, M. A., Baumgartner, J. v. AND olson, E. C. 1985 b. Patterns of temporal change in single morphological characters of a Miocene stickleback fish. Paleobiology 11, 258-271.

CAMPBELL, R. N. 1979. Sticklebacks [Gasterosteus aculeatus (L.) and Pungitius pungitius (L.)] in the Outer Hebrides, Scotland. Hebridean Naturalist, 3, 8-15.

COAD, B. W. 1973. Modifications of the pelvic complex in ninespine sticklebacks, Pungitius pungitius (L.), of eastern Canada and the Northwest Territories. Naturaliste Canadien, 100, 315-316.

EDGE, T. A. AND COAD, B. W. 1983. Reduction of the pelvic skeleton in the threespine stickleback, Gasterosteus aculeatus, in two lakes of Quebec. Canadian FieldNaturalist, 97, 334-336.

FALCONER, D. S. 1965. The inheritance of liability to certain diseases, estimated from the incidence among relatives. Annals of Human Genetics, 29, 51-76.
FALCONER, D. S. 1981. Introduction to Quantitative Genetics. 2nd edn, Longman, London.

GILES, N. 1983. The possible role of environmental calcium levels during the evolution of phenotypic diversity in Outer Hebridean populations of the threespine stickleback, Gasterosteus aculeatus. Journal of Zoology, London, 190, 535-544.

HAGEN, D. W. 1973. Inheritance of numbers of lateral plates and gill rakers in Gasterosteus aculeatus. Heredity, 30, 303-312.

HOOGLAND, R. D., MORRIS, D. AND TINBERGEN, N. 1957. The spines of sticklebacks (Gasterosteus and Pungitius) as means of defense against predators (Perca and Esox). Behaviour, 10, 205-230.

LANDE, R. 1978. Evolutionary mechanisms of limb loss in tetrapods. Evolution, 32, 73-92.

MCPHAIL, J. D. 1963. Geographic variation in North American ninespine sticklebacks, Pungitius pungitius. Journal of the Fisheries Research Board of Canada, 20, 27-44.

MCPHAIL, J. D. (in press). Speciation and evolution of reproductive isolation in the sticklebacks (Gasterosteus) of southwestern British Columbia. In: Bell, M. A. and Foster, S. (eds). Evolutionary Biology of the Threespine Stickleback, Oxford University Press, Oxford.

MOODIE, G. E. E. AND REIMCHEN, T. E. 1973. Endemism and conservation of sticklebacks in the Queen Charlotte Islands. Canadian Field Naturalist, 87, 173-175.

NELSON, J. S. 1969. Geographic variation in the brook stickleback, Culaea inconstans, and notes on nomenclature and distribution. Journal of the Fisheries Research Board of Canada, 26, 2431-2447.

NELSON, J. S. 1971a. Comparison of the pectoral and pelvic skeletons and some other bones and their phylogenetic implications in the Aulorhynchidae and Gasterosteidae (Pisces). Journal of the Fisheries Research Board of Canada, 28, 427-442.

Nelson, J. S. 1971b. Absence of the pelvic complex in ninespine sticklebacks, Pungitius, collected in Ireland and Wood Buffalo National Park Region, Canada, with notes on meristic variation.Copeia, 1971, 707-717.

NELSON, J. S. 1977. Evidence of a genetic basis for absence of the pelvic skeleton in brook stickleback, Culaea inconstans, and notes on the geographical distribution and origin of the loss. Journal of the Fisheries Research Board of Canada, 34, 1314-1320.

NELSON, J. S. AND ATTON, F. M. 1971. Geographic and morphological variation in the presence and absence of the pelvic skeleton in the brook stickleback, Culaea inconstans (Kirtland), in Alberta and Saskatchewan. Canadian Journal of Zoology, 49, 343-352.

PALMER, A. R. AND STROBECK, C. 1986. Fluctuating asymmetry: measurement analysis, patterns. Annual Review of Ecology and Systematics, 17, 391-421.

REIMCHEN, T. E. 1980. Spine deficiency and polymorphism in a population of Gasterosteus aculeatus: an adaptation to predators? Canadian Journal of Zoology, 58, 1232-1244. REIST, J. D. 1980a. Predation upon pelvic phenotypes of brook stickleback, Culaea inconstans, by selected invertebrates. Canadian Journal of Zoology, 58, 1253-1258. 
REIST, J. D. 1980b. Selective predation upon pelvic phenotypes of brook stickleback Calaea inconstans, by northern pike, Esox lucius. Canadian Journal of Zoology, 58, 1245-1252.

REIST, J. D. 1981. Variation in frequencies of pelvic phenotypes of the brook stickleback, Culaea inconstans, in Redwater drainage, Alberta. Canadian Field-Naturalist, 95, 178182.

TAKATA, K AND GOTO, A. 1985. Pelvic spine deficiency of
Pungitius tymensis in Hokkaida. Japanese Journal of Ichthyology, 32, 100-103.

STEPHANidis, A. 1971. About some of the fishes of the freshwater of Greece. Biologia Gallo-Hellenica, 3, 213-241. wootton, R. J. 1976. The Biology of The Sticklebacks. Academic Press, New York.

wootton, R. J. 1984. A Functional Biology of Sticklebacks. Croom Helm, London. 